\title{
Geospatial Analysis of Statin Adherence Using Pharmacy Claims Data in the State of Michigan
}

\author{
Steven R. Erickson, PharmD, and Yuan-Nung Tony Lin, PharmD
}

\section{ABSTRACT}

BACKGROUND: Research has demonstrated that variation in availability and utilization of health care resources exist on a range of scales, from regions of the United States, hospital referral regions, ZIP codes, and census tracts. Limited research using spatial analyses has found that variation in medication adherence exists across census tracts. Using spatial analysis, researchers may be able to effectively analyze geographically dispersed data to determine whether factors such as sociodemographics, local shared beliefs and attitudes, barriers to access such as availability of prescribers or pharmacies, or others are associated with variations in medication adherence in a defined geographic area.

OBJECTIVES: To (a) demonstrate that medication adherence may be mapped across an entire state using medication possession ratios and (b) determine whether a geographic pattern of adherence to statins could be identified at the ZIP code level for members of a statewide insurer.

METHODS: This study utilized pharmacy claims data from a statewide insurer. Insured statin users were aged $>\mathbf{3 0}$ years, had at least 1 statin prescription, and were continuously enrolled for the observation year. Patient medication possession ratios (MPR) were derived, which were then aggregated as a mean MPR for each ZIP code. ZIP codes were categorized as higher (MPR $>0.80$ ) or lower (MPR $<0.80$ ) adherence and mapped using Arc GIS, a platform for designing and managing solutions through the application of geographic knowledge. Analysis included a determination of whether the MPRs of higher and lower adherence ZIP codes were significantly different. Hot spot analysis was conducted to identify clustering of higher, midrange, and lower adherent ZIP codes using the GetisORD Gi* Statistic. This test provides z-scores and $P$ values to indicate where features with either high or low values cluster spatially. MPRs for these 3 categories were compared using one-way analysis of variance (ANOVA).

RESULTS: Of 1,154 Michigan ZIP codes, 907 were represented by 212,783 insured statin users. The mean statin MPR by ZIP code was $0.79 \pm 0.4$. The mean MPR for higher adherent ZIP codes was $0.83 \pm 0.03$ and $0.76 \pm 0.03$ for lower adherent ZIP codes $(P<0.001)$. Significant clustering of ZIP codes by adherence levels was evident from the hot spot analysis. The mean MPR was $0.84 \pm 0.04$ for high adherence areas, $0.79 \pm 0.03$ for midrange areas, and $0.74 \pm 0.04$ for lower adherent areas (overall $P<0.001$ ).

CONCLUSIONS: Significant variations in adherence exist across ZIP codes at a state level. Future research is needed to determine locally relevant factors associated with this finding, which may be used to derive locally meaningful interventions.

J Manag Care Pharm. 2014;20(12):1208-15

Copyright $\odot 2014$, Academy of Managed Care Pharmacy. All rights reserved.

\section{What is already known about this subject}

Medication adherence is associated with numerous patient, disease, and treatment variables. Variables that are associated with medication adherence tend to be patient-centered or patientspecific.

Performance of health-related behaviors such as diet and exercise have been shown to be associated not only with patient factors, but also geographic or neighborhood factors.

Previous research has demonstrated that self-reported adherence is a behavior that can be mapped and that variation does exist geographically at the census tract level as well as the much larger hospital referral level.

\section{What this study adds}

Adherence to a class of cholesterol-lowering drugs, or statins, varies geographically at the ZIP code level throughout an entire state. Mapping adherence of a managed care population may be a useful tool to identify geographic areas of lower adherence. There may be local population-based reasons for lower adherence, which may be investigated and incorporated into adherenceimproving interventions.

T he World Health Organization defines "medication adherence" as the extent to which a patient's behaviortaking medication, following a diet, and/or executing lifestyle changes - corresponds with agreed recommendations from a health care provider. ${ }^{1}$ Medications are unlikely to lead to optimal outcomes if they are not taken appropriately; therefore, adherence is the primary determinant of therapy success.

A commonly prescribed class of medications used to lower cholesterol is called statins. When a patient is adherent to a prescribed statin therapy, the number of cardiac events related to acute coronary syndrome is reduced by as much as $60 \%$, and risk of stroke is reduced by $17 \%$ after long-term treatment. ${ }^{2}$ However, nonadherence may lead to negative outcomes, as was demonstrated in a study of patients with coronary artery disease. Nonadherence was associated with increased risk of cardiovascular-related adverse events and resource utilization. ${ }^{3}$ Many factors have been found to contribute to medication nonadherence, including a patient's perception of the disease; ${ }^{4-6}$ age, cultural, and ethnic background $;{ }^{7}$ socioeconomic status; ${ }^{8}$ complexity of the drug regimen; ${ }^{9}$ and access to medical care. ${ }^{8}$ 
It is estimated that only approximately half of patients with chronic disease adhere to their treatment recommendations. ${ }^{1}$

Two other factors that may be associated with medication adherence, but not extensively studied to date, are population characteristics and neighborhood effects. The term "neighborhood effect" is used to describe the social interaction that influences the behavior or socioeconomic outcome of an individual. ${ }^{10}$ It has recently been used to describe factors associated with health status. ${ }^{11}$ Clinicians and researchers are partnering to examine the association between the environment in which people live and the development of illness, as well as to identify modifiable risk behaviors in populations. For example, individuals living in lower socioeconomic areas are at greater risk of developing cardiovascular disease, and those living in deprived neighborhoods have lower survival rates after a myocardial infarction. ${ }^{12,13}$ Social characteristics of a local environment are associated with residents participating in health-promoting behaviors. ${ }^{14}$

A recent study demonstrated the feasibility of using a software program that not only can map geocoded variables but also has the ability to analyze data based on location. ${ }^{15}$ This study used self-reported medication adherence as a dependent variable, which was then mapped to a patient's home address and analyzed at the census tract level. The analysis demonstrated that adherence to 5 classes of medications used by patients after experiencing an acute coronary syndrome event varied across a defined geographic area.

On a larger scale, adherence to hypoglycemic medications by patients with diabetes varies by U.S. region. ${ }^{16}$ Another study demonstrated that use of medications varies geographically, focusing not on patient adherence as an outcome, but on prescription patterns, using hospital referral regions as the geographic unit of measure. ${ }^{17}$ Medications prescribed for Medicare enrollees for the treatment of diabetes varied by region of the country, with prescription patterns associated with household income and race. A more recent study analyzed the association between individual and neighborhood variables with viral load as a proxy measure for adherence to prescribed antiretroviral therapies for patients with human immunodeficiency virus infection. ${ }^{18}$ Patients living in neighborhoods with higher rates of unemployment were less likely to have a current antiretroviral prescription.

Assessing medication adherence at the population level may provide multidimensional analysis that includes not only individual characteristics but also contextual factors found in the patient's environment and social network. ${ }^{19}$ We hypothesized that medication-taking behavior will vary across the state of Michigan in a group of patients prescribed statin medications that have prescription medication insurance. The objectives of this study were twofold. The first objective was to demonstrate that medication adherence may be mapped across an entire state. Medication possession ratios were derived from a pharmacy claims dataset for statin drugs obtained from a large insurer with a statewide presence. Our second objective was to determine whether there was clustering of ZIP codes into areas of higher or lower adherence. Identifying areas of high and low adherence may lead analysts to further attempt to identify shared facilitators or barriers to adherence that may be locally relevant. This information may then be used by health plans and other providers to design and tailor interventions to target and mitigate the effects of the previously described factors on medication adherence with the hope of improving therapeutic outcomes.

\section{Methods}

\section{Study Design}

This cross-sectional study used pharmacy administrative claims data from July 1, 2009, to June 30, 2010, to calculate medication possession ratios (MPR) of statin drug use for patients with prescription drug insurance from the largest health care insurance provider in Michigan. The insurance company's portfolio of plans have over $50 \%$ of the market share in the state. ${ }^{20}$

\section{Subjects}

Data were obtained from a large health insurer with enrollees throughout Michigan that includes prescription drug plans in its business portfolio. A data use agreement was signed by both parties prior to obtaining the data. The study was approved by the University of Michigan's Human Subjects Review Board. The insurer did not provide data based on patient home addresses or at the census tract level due to concerns for patient privacy. Claims data for statin medication were linked to patient home ZIP codes. This prevented analysis at smaller geographic units such as census tract or census block.

The insurer constructed the dataset that provided patientspecific data, including the patient's age and gender, along with all statin prescription fill and refill information for the study period from July 1, 2009, to June 30, 2010 (drug name and number of doses dispensed). The geocode link for each patient in the dataset was the ZIP code associated with the home address. It was assumed that patients in the dataset lived at the listed home address during the entire study period.

Subjects selected for the insured statin user group were continuously enrolled with prescription drug coverage for a full year starting July 1, 2009, and also had at least 1 prescription filled (pharmacy claim) for any statin drug during the study period. The reason for choosing 1 prescription fill versus 2 or more as a threshold is that with statin therapy, once the decision is made to start, it is typically a long-term commitment. Subjects must also have had a home address ZIP code listed in the insurer's database that coincided with ZIP codes found within the state. Subjects had to be at least aged 30 years or older and less than aged 88 years, which is the upper age limit 
that the insurer would identify patients based on its patient confidentiality rules. The age of 30 was chosen as the lower threshold age based on the notion that cardiovascular disease begins to appear in males starting at this age, increasing the likelihood of being on a statin. ${ }^{21}$ Subjects were excluded if they were institutionalized (e.g., living in a nursing home). Age and gender were used to describe the insured statin user sample. Data were then aggregated at the ZIP code level. For this study, the insured statin user, or enrollee, is referred to as the patient.

\section{Medication Possession Ratio}

For this study, MPR was used to determine medication adherence over other methods to characterize adherent behavior because of the simple nature of the statin drug regimen-1 drug taken once daily. For simple regimes such as statin therapy, MPR is found to be a valid measure. ${ }^{22}$ The MPR was derived by determining the fill date for the first prescription in the study period, which is determined by the date the pharmacy submits a claim for a statin prescription to the insurer. This date became the start of the individual patient's study period. The time, measured in days, between the first fill date of the statin medication and June 30,2010, was used as the denominator in determining the MPR. The denominator represents the total possible number of days the patient should be taking the medication. An assumption was made that once statin drug therapy is started, it should be continued on a longterm daily basis.

The numerator in the MPR is the actual number of days, or in the case of statins, the number of tablets dispensed based on pharmacy claims during the study period up to June 30 , 2010. For example, if a patient had a prescription filled on July 1, 2009, for 90 tablets, and had 3 additional prescriptions of 90 tablets filled during the year, the MPR for this patient would be $360 / 365$, or 0.986 . This equates to almost perfect adherence/ persistence. Another example would be a patient who filled the first prescription for statin therapy on February 1, 2010, for 90 tablets and had no other refills during the study period based on pharmacy claims. The MPR for this patient would be approximately $90 / 150$ or 0.6 . This equates to a patient being adherent to the statin prescription $60 \%$ of the potential time the patient could take the medication.

The MPR was determined for all statin prescriptions filled by a patient during the study period. If a drug regimen contained overlap when 1 statin was changed to a different statin drug midway between a refill of the original statin agent, the overlap was deleted from the calculation of the numerator. In that way, adherence was not overinflated, making the assumption that the patient would not continue to take an old prescription and would instead discard the old product and start the new drug regimen.

Statin drugs included in this study were simvastatin, pravastatin, rosuvastatin, lovastatin, fluvastatin, and atorvastatin. It is feasible that some patients who were initiated on statin therapy during the reference time study period were changed to a nonstatin drug due to reasons such as adverse effects to the drugs. Other potential limitations to using pharmacy claims data to determine medication adherence would include situations where patients pay cash rather than use their insurance or have access to a secondary plan, which may pay for the medication, to name a few. To be included in this study, the patient must have been insured during the study period, which would preclude including patients who dropped the insurance plan during the study period. The investigators acknowledge that there are limitations to using pharmacy claims data to determine medication adherence and persistence.

ZIP codes were excluded from analysis if the total number of patients living in that ZIP code was less than 10 in order to reduce the possible bias that may be introduced into analysis by having too few patients in a ZIP code.

\section{Geographic Information System}

A geographic information system (GIS) integrates hardware, software, and data to allow a user to store, analyze, manipulate, and present geographically referenced information. The $3 \mathrm{com}-$ ponents to a GIS include a structured database, map generator, and analysis programming to determine spatial relationships between variables. The software used for this study was Arc GIS 10.1 by Esri (Redlands, CA).

The dataset obtained from the insurer included the home residence ZIP code of the insured statin user. The ZIP code and related data for each patient were then mapped in a process known as geocoding. Geocoding mapped each patient's ZIP code to geographic coordinates, and that information was then aggregated for each ZIP code. Demographic information and patient adherence data (MPRs) were also aggregated at the ZIP code level for analysis.

\section{Analysis}

Descriptive analyses of demographics and the adherence data (MPRs) were presented as means and standard deviations for continuous variables or frequencies and percentages for categorical variables. The MPRs of all patients residing within the same ZIP codes were averaged to derive an MPR average for the ZIP code.

In order to meet the first study objective, the mean MPR for each ZIP code was calculated and assigned a label of high adherence if the value was $\geq 0.80$ or lower adherence if the MPR value was $<0.80$. The threshold of 0.80 used to determine adherent versus nonadherent groups is commonly used in adherence research and has some justification based on outcomes. ${ }^{23}$ A map was then created of Michigan ZIP codes based on lower or higher adherence levels (Figure 1).

To evaluate the second study objective, hot spot analysis was conducted to determine groupings of ZIP codes with similar 


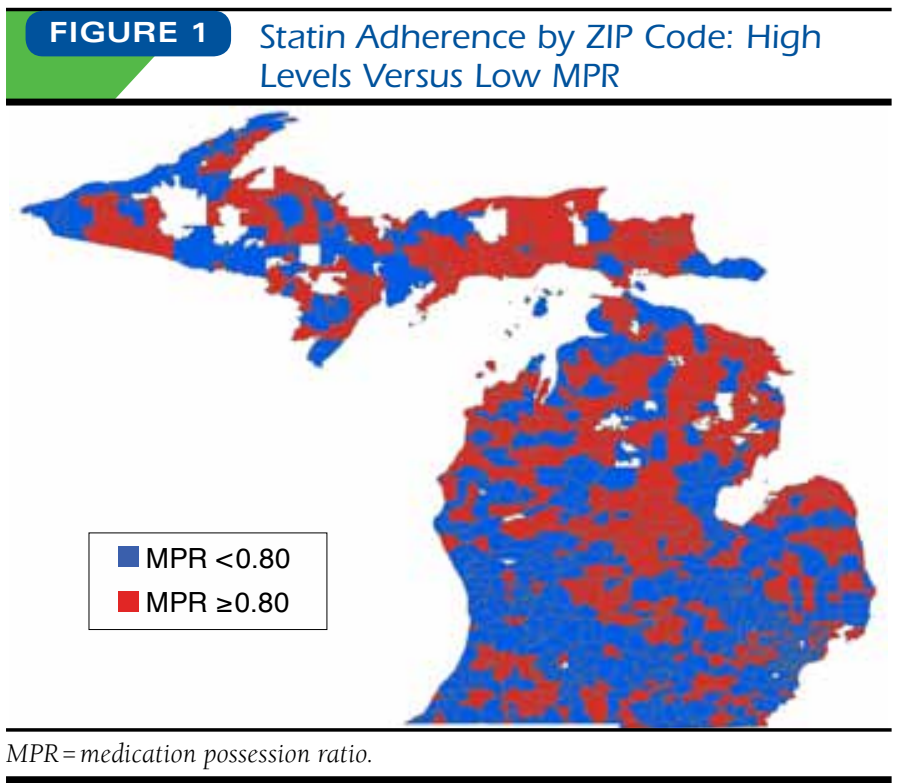

MPR areas of high and low adherence (nearest neighbor or polygon contiguity analysis). Polygon contiguity analysis is effective when polygons (for this study, ZIP codes) are similar in size and distribution and when spatial relationships are a function of polygon proximity. This method contrasts the MPRs of directly adjacent ZIP codes with the target ZIP code of each calculation.

The Getis-ORD Gi* statistic was calculated for each ZIP code, resulting in a $z$-score and $P$ value. The $z$-score informed us where high/low MPR values are clustered spatially (i.e., ZIP codes that are similar in adherence to their bordering neighbors). A statistically significant hot spot had a high value that is surrounded by other features with high values, where the $z$-score is high and the $P$ value is small. A low negative $z$-score and a small $P$ value indicates a significant cold spot. The local sum for a ZIP code and its neighbors was compared proportionally with the sum of all ZIP codes. When the local sum was much different than the expected sum (local), meaning the magnitude of difference was too great to be due to chance, a statistically significant $z$-score resulted.

The hot spot analysis tool in Arc GIS assessed whether high/ low values cluster spatially. The larger the z-score, the more intense the clustering of high values (hot spot). The lower the $z$-score, the more intense the clustering of low values (cold spot). In this study, hot spots relate to better adherence, while cold spots refer to lower adherence values. A ZIP code with a z-score that is neither high nor low is considered neutral (midrange)not statistically significantly different from its neighbors.

A map was derived from this analysis, consisting of 7 levels of adherence based on the z-scores assigned to the individual ZIP codes from the hot spot analysis (Figure 2). Hot spots are

\section{FIGURE 2 Statin Adherence by Hot Spot Analysis}

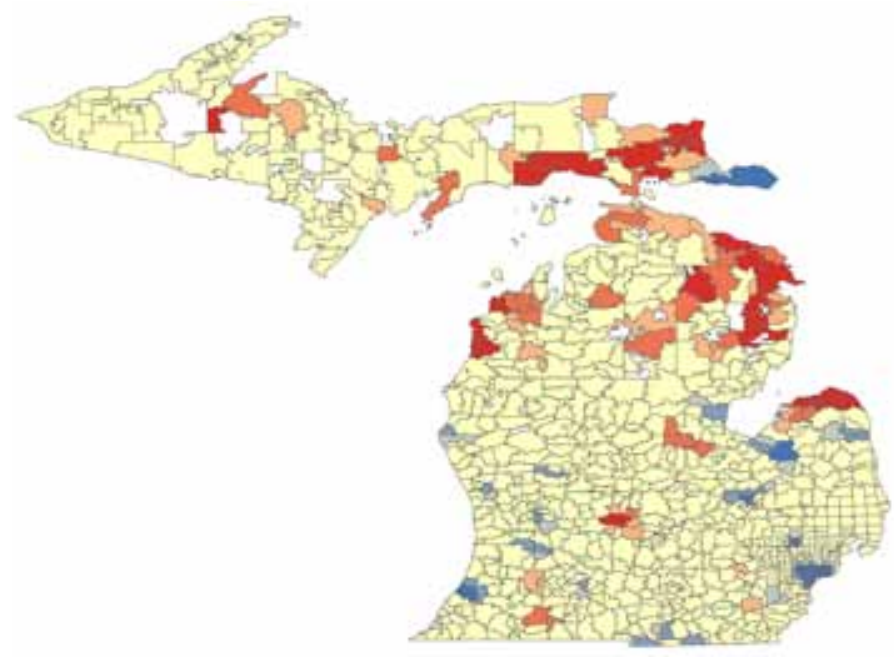

Cold Spot $(99 \% \mathrm{Cl})$, mean MPR $0.72(0.03)$

Cold Spot $(95 \% \mathrm{Cl})$, mean MPR $0.75(0.04)$

Cold Spot $(90 \% \mathrm{Cl})$, mean MPR $0.75(0.03)$

Not Significant (neutral, midrange), mean MPR 0.79 (0.03)

Hot Spot $(90 \% \mathrm{Cl})$, mean MPR $0.82(0.04)$

Hot Spot $(95 \% \mathrm{Cl})$, mean MPR $0.84(0.04)$

Hot Spot $(99 \% \mathrm{Cl})$, mean MPR $0.85(0.04)$

$C I=$ confidence interval $; M P R=$ medication possession ratio

denoted on the map in 3 shades of red, with darkest red being z-score of 99\% confidence interval (CI; highest adherence), medium red with a $z$-score of 95\% CI, and light red being 90\% CI. ZIP codes that showed no significant differences in MPRs between bordering ZIP codes are considered neutral ZIP codes (midrange) and are denoted by the color yellow. Cold spots are represented in shades of blue, with the darkest blue representing ZIP codes with 99\% CI (lowest adherence), medium blue with 95\% CI, and light blue with 90\% CI. Analysis using oneway ANOVA, with the Scheffe test for post hoc analysis, of the mean MPR for each of the 7 adherence categories derived from the hot spot analysis demonstrated a grouping of 3 categories of adherence (higher, neutral or midrange, and lower). These categories were further analyzed using one-way ANOVA to generate the mean and standard deviation of the MPR for each of the 3 categories.

\section{Results}

MPRs were calculated for a total of 214,966 patients using statins in 1,136 of the 1,154 total ZIP codes in Michigan. Of these, 907 ZIP codes, which included 212,783 patients, had 10 or more patients residing within their boundaries. These ZIP codes were used for subsequent analyses. The mean number of patients per ZIP code used for analysis ranged from 10 to 
1,389 patients, with a mean of $234.6 \pm 259.5$. The mean patient age was $59.4 \pm 2.7$ years, with $43.2 \%$ being female. The overall mean statin MPR by ZIP code was $0.79 \pm 0.04$, with a range of 0.59 to 0.95 . Lower MPR is indicative of lower adherence.

Figure 1 provides a visual depiction using the map showing the distribution of ZIP codes with higher (MPR $\geq 0.80$, red color) or lower (MPR $<0.80$, blue color) adherence based on statin MPR. A total of 366 (40.4\%) ZIP codes had an average MPR of 0.80 or higher (higher adherence), while 541 (59.6\%) ZIP codes had an average MPR of less than 0.80 (lower adherence). The mean MPR for higher adherence ZIP codes was $0.83 \pm 0.03$, while the mean MPR for lower adherence ZIP codes was $0.76 \pm 0.03(P<0.001)$. The mean age of patients residing in higher adherence ZIP codes was $60.0 \pm 0.3$ years and $59.0 \pm 2.7$ years for the lower adherence ZIP codes $(P<0.001)$. The percentage of patients who were female was $42.8 \%$ for higher adherence ZIP codes and $43.4 \%$ for lower adherence ZIP codes $(P=0.18)$.

Figure 2 is a map of the hot spot analysis. Significant clustering of ZIP codes by adherence levels is evident. The majority of ZIP codes are yellow ( $80.2 \%$ of 727 ). The mean MPR for the yellow ZIP codes was $0.79 \pm 0.03$. The hot spots consisted of 3 levels of MPR, with the most adherent (darkest red) having a mean MPR of $0.85 \pm 0.04$ for 30 ZIP codes; medium red having a mean MPR of $0.84 \pm 0.04$ with 27 ZIP codes; and the lightest red with an average MPR of $0.82 \pm 0.04$ for 33 ZIP codes. The cold spots consisted of 3 levels of MPR, with the least adherent (darkest blue) having a mean MPR of $0.72 \pm 0.03$ for 38 ZIP codes; medium blue with a mean MPR of $0.75 \pm 0.04$ for 28 ZIP codes; and light blue with a mean MPR of $0.75 \pm 0.05$ for 24 ZIP codes.

Looking at the entire state map, hot spots (higher adherence) tended to be in the upper or northern ZIP codes of the state, while the lower portion of the state had a mix of hot (higher adherence) and cold spots (lower adherence). The greatest concentration of cold spots was in the metropolitan area of Detroit in the lowest southeastern part of the state; to the north of metropolitan Detroit in the Flint and Saginaw/ Bay City area; and to the far lower west side in the St. Joseph/ Benton Harbor area. These areas represent many of the urban/ suburban centers of the state. Other cold spot areas were found in less populated areas, as well along the bottom of the state and on both east and west coasts. One standout cold spot is on the eastern end of the Upper Peninsula. The ZIP codes with highest adherence (dark red) contain populated areas of smaller cities and townships as well as less populated areas. The larger metropolitan Grand Rapids area (midwestern section of the state) tended to be neutral to lower adherence, and the Lansing metropolitan area (middle lower portion of the state) showed neutral to higher adherence.
The result of the post hoc analysis using the Scheffe test for the one-way ANOVA of the 7 levels of MPR demonstrated that there were 3 groupings of MPRs: (a) all 3 cold spot categories (lower adherence), (b) the single neutral (midrange) category, and (c) all 3 hot spots (higher adherence). These 3 categories were then further analyzed using one-way ANOVA. The high adherence category had a mean MPR of $0.84 \pm 0.04$ for high adherence areas $(n=90) ; 0.79 \pm 0.03$ for neutral areas $(n=727)$; and $0.74 \pm 0.04$ for lower adherent areas $(n=90 ; P<0.001)$.

\section{Discussion}

This study demonstrates that it is feasible to map medicationtaking behavior of patients using statin therapy. According to our findings, variations in adherence exist across ZIP codes at a statewide level. The 2 spatial analyses provided an incremental examination of the patterns of medication-taking behavior with visual inspection of the 2 maps showing concordant patterns.

While the data presented in Figure 1 are a simple assessment of adherence based on higher or lower adherence based on MPR dichotomized at 0.80 , the results of the hot spot analysis in Figure 2 provide a smoothing technique to allow for assessment of clustering of high and low MPRs that represent extremes in medication-taking behavior. Significant clustering of ZIP codes by adherence levels is evident, with lower adherence concentrated in several metropolitan areas in Michigan, including the Detroit metropolitan area in southeastern Michigan, the Flint metropolitan area in eastern midMichigan, and the St. Joseph/Benton Harbor metropolitan area on the lower west side of the state. Areas of higher adherence were noted in northern Michigan, the tip of the "thumb" of the lower peninsula, and in the smaller metropolitan areas of Lansing and Kalamazoo.

One issue to note is that the hot spot analysis may smooth over areas where the difference between neighboring ZIP codes is not significant, which may mask some areas where adherence is a problem. An example of this may be observed at the western edge of Michigan's upper peninsula, where, in Figure 1, the ZIP codes have a mean MPR below 0.80 (lower adherence), but in the hot spot analysis, the same ZIP codes are not significantly different from their neighboring ZIP codes.

Demographic variables have been associated with adherence in previous studies. Through a systematic review, Daley et al. (2012) found several demographic factors associated with nonadherence, including younger age and low income. ${ }^{24}$ Grosset et al. (2005) showed that increased age was associated with better overall adherence, ${ }^{25}$ which is consistent with our findings that patients with higher adherence were older than patients with lower adherence. Of note, however, is that the age difference between the high and low adherent groups in our study was only 1 year, although it was statistically significant. Other commonly assessed demographic variables such as 
household income and level of education were not assessed in our study due to the lack of this information in the administrative claims data. It is important to remember that the patients used in this study had prescription insurance that covered the statin medications analyzed in this study. Having insurance with lower copays has been shown to be associated with better adherence compared with higher copays. ${ }^{26}$ We did not have information on copay for each patient, so this variable could not be assessed. However, having prescription insurance is associated with better adherence. ${ }^{27}$

This study used variables that were obtained from a prescription insurance administrative database to derive independent and dependent variables. More detailed self-reported data about patients' beliefs and attitudes toward taking medication were not obtained. It is reasonable to hypothesize that other social, behavioral, or resource-related factors may be associated with geographic variation in medication adherence. The concept that a "neighborhood effect" may be associated with medication adherence is relatively new. A neighborhood effect includes the association between the physical environment, social networking and interactions, and other effects within a geographic area. ${ }^{10,11,28,29}$

Neighborhood or local effects have been demonstrated to be associated with health outcomes associated with health behaviors. Health behaviors such as exercise, diet, and taking medication are associated, to varying degrees, with social network factors that occur within neighborhoods as well as beyond these confined geographic areas. ${ }^{30,31}$ A recent analysis of the spread of obesity in a large social network over 32 years found that clusters of obese patients were identifiable, with clusters extending to 3 degrees of separation. Chances of becoming obese increased $57 \%$ if patients had friends who became obese during a given period. However, these effects were not seen in an immediate geographic location, such as a neighborhood, but rather through social connections. ${ }^{32}$ Other studies have found that lack of participation, integration, and connection with social networks are associated with negative health outcomes $^{33,34}$ and medication nonadherence. ${ }^{35}$ Conversely, social interactions may have unintentional negative influences on self-management behavior. Because of misconceptions or lack of understanding, friends and family members may influence a patient's behavior in unsupportive or inappropriate ways, such as offering well-intentioned advice that conflicts with a physician's self-management recommendations or directly or indirectly promotes unhealthy behaviors. ${ }^{34}$ According to Chaix's review (2009), associations have been found to exist between an individuals' social support and coronary heart disease (CHD) ${ }^{36}$ It is suggested that any eco-epidemiologic model of $\mathrm{CHD}$ and its risk factors should consider social interactions and the physical environment.
The study of geographic variation in prevalence of illness, the use of health care services, and measurement of health-related outcomes have provided important information to clinicians, policymakers, and researchers for a wide range of conditions and treatments. For example, in cardiovascular medicine, significant geographic variation exists in performance of carotid revascularization procedures among Medicare beneficiaries, ${ }^{37}$ physician propensity to treat specific cardiovascular conditions,$^{38}$ the adherence to specific diet and prevalence of acute coronary syndromes, ${ }^{39}$ and incidence and outcomes of patients hospitalized for myocardial infarction. ${ }^{40}$ On a more local level, examples of studies of the association between neighborhood effects and cardiovascular-related outcomes include the effect of neighborhood of residence and incidence of coronary heart disease ${ }^{41}$ neighborhood characteristics and bystander-initiated cardiopulmonary resuscitation, ${ }^{42}$ and survival after experiencing a myocardial infarction. ${ }^{13,43}$

Examples of studies of geographic variation in utilization of medications include a range of topics, such as diabetes medications,${ }^{17}$ antiretroviral treatment among Medicaid enrollees,$^{44}$ opiate medication use in state Medicaid fee-for-service programs, ${ }^{45}$ and opioid use by patients who have fibromyalgia. ${ }^{46}$ GIS software programs, which have the ability to map data and perform spatial analyses, are a relatively new tool used to assess geographic variation in medication utilization by populations. Czarnecki et al. (2010) utilized GIS to track and analyze patterns of nicotine replacement therapy utilization provided by the New York City Department of Health and Mental Hygiene. ${ }^{47}$ Analysts were able to demonstrate that in neighborhoods with high smoking prevalence, the lower income neighborhoods had higher enrollment in the nicotin replacement program compared with higher income neighborhoods. The study also demonstrated that GIS analysis was able to detect the effect of a press release advertising the availability of the nicotine replacement program to the public. Brownstein et al. (2010) used GIS software to map and analyze patient data from addiction treatment facilities in New Mexico to understand the spatial distribution of opioid abuse at the local level..$^{48}$ Investigators found clustering of abuse around a metropolitan area and that drug availability was partly responsible for the clustering of prescription opioid abuse.

GIS software and spatial analysis was used in a study of geographic variation of self-reported medication adherence of patients who were discharged from a large academic health system after treatment for acute coronary syndrome (ACS). ${ }^{15}$ Using data from the health system's ACS registry, self-reported medication adherence was mapped to patients' home addresses and analyzed at the census tract level. The analysis demonstrated that adherence to 4 classes of medications used by patients after experiencing an ACS event varied across a defined geographic area, with census tracts located in and around the city where the health system is located having higher adherence compared with outlying areas. 
The present study further demonstrates the ability to identify spatial patterns of adherent and nonadherent medicationtaking behavior, this time at the ZIP code level throughout the state of Michigan. This finding alone can inform public health professionals about varying levels of adherence geographically. Future studies can utilize the same approach to identify areas of low adherence, coupled with community-based qualitative studies to identify locally relevant barriers to adherent behavior. Ideally, from these studies will come interventions that are community-specific and geographically relevant, depending on the types of barriers to adherence that may be identified. Whether low income, low levels of education, physical environment, locally entrenched beliefs and attitudes, or various sociodemographic characteristics that are associated with lower adherence, specific tailored interventions may be developed to target a geographic area, such as neighborhood or ZIP code. This could be more effective and more efficient than advertising a general message to a population or individual patients.

\section{Limitations}

A limitation of this study is that it was assumed that the insured statin users were adherent if they refilled their statins on time. Although a logical assumption, we cannot be completely certain that all medication was consumed as directed between refill dates. This means the MPRs calculated in this study could sometimes be overestimating adherence. In addition, the patients could have moved to a different ZIP code from what was recorded in the dataset. Another limitation is that all the patients whose data were used to calculate the MPRs had prescription drug coverage. It is quite possible that these insured statin users varied in sociodemograhics, as well as beliefs and attitudes about taking medication, from other people living in the same ZIP code who either have different prescription insurance or no insurance coverage at all. Further research should include patients with variations in types of prescription drug coverage as well as those without coverage. The adherence level for patients with insurance may be better than those who do not have insurance due to reduced barriers related to drug cost. This may depend on copays and if pharmacies offer discounted drug programs. There may actually be poorer adherence in ZIP codes where more patients lack prescription drug coverage compared with other areas, and this difference may not be observed with the current study. Another limitation is that some patients may actually pay cash and bypass the prescription adjudication process if they utilize discount generic programs offered by many pharmacies throughout Michigan. If this happens, there may be artificial gaps in the pharmacy claims data that may lead to artificially low MPRs (lower adherence). The extent of this practice is not known in the insured population used for this study.

\section{Conclusions}

It is possible to visually map the statin medication adherence for an insured population in a state. As shown in this study, variations in adherence existed across ZIP codes, with areas of lower adherence being found in ZIP codes associated with larger metropolitan areas as well as a number of more rural ZIP codes. Higher adherence was found more often in ZIP codes in the more rural upper portions of the state as well as in and around several smaller metropolitan areas. Future research is needed to determine local, neighborhood, and contextual variables that may be associated with these findings. Implications for managed care are to identify areas of lower adherence, determine locally relevant reasons that may be associated with lower adherence, and develop interventions to remove barriers to mitigate the effect of the factors involved.

\section{Authors}

STEVEN R. ERICKSON, PharmD, is Associate Professor, University of Michigan College of Pharmacy, Ann Arbor, Michigan, and YUAN-NUNG TONY LIN, PharmD, is Staff Pharmacist, Diplomat Pharmacy, Flint, Michigan.

AUTHOR CORRESPONDENCE: Steven R. Erickson, PharmD, Associate Professor, University of Michigan College of Pharmacy, 428 Church St., Ann Arbor, MI 48109-1065. Tel.: 734.763.4989; Fax: 734.763.2022; E-mail: serick@med.umich.edu.

\section{DISCLOSURES}

Neither author has any financial interest or affiliation with any company, product, or service described in the manuscript. This study was funded by the University of Michigan Cardiovascular Center Microgrant program. At the time of the study, Lin was a pharmacy student at the University of Michigan College of Pharmacy.

Erickson was primarily responsible for study concept and design, data collection and interpretation, and writing and revising of the manuscript, along with Lin.

\section{REFERENCES}

1. World Health Organization. Adherence to long-term therapies: evidence for action. 2003. [Page 28]. Available at: http://www.who.int/chp/knowledge/ publications/adherence_introduction.pdf. Accessed October 6, 2014.

2. Law MR, Wald NJ, Rudnicka AR. Quantifying effect of statins on low density lipoprotein cholesterol, ischaemic heart disease, and stroke: systematic review and meta-analysis. BMJ. 2003;326:1423-29.

3. Ho PM, Magid DJ, Shetterly SM, et al. Medication nonadherence is associated with a broad range of adverse outcomes in patients with coronary artery disease. Am Heart J. 2008;155(4):772-79.

4. Jin J, Sklar GE, Min Sen Oh V, Chuen Li SC. Factors affecting therapeutic compliance: a review from the patient's perspective. Ther Clin Risk Manag. 2008;4(1):269-86

5. Khanderia U, Townsend KA, Erickson SR, Vlasnik J, Prager RL, Eagle KA. Medication adherence following coronary artery bypass graft surgery: assessment of beliefs and attitudes. Ann Pharmacother. 2008;42(2):192-99.

6. Phatak HM, Thomas J. Relationships between beliefs about medications and nonadherence to prescribed chronic medications. Ann Pharmacother. 2006;40(10): 1737-42. 
7. Mann DM, Allegrante JP, Natarajan S, Halm EA, Charlson M. Predictors of adherence to statins for primary prevention. Cardiovasc Drugs Ther. 2007;21(4):311-16.

8. Avorn J, Monette J, Lacour A, et al. Persistence of use of lipid-lowering medications: a cross-national study. JAMA. 1998;279(18):1458-62.

9. Choudhry NK, Fischer MA, Avorn J, et al. The implications of therapeutic complexity on adherence to cardiovascular medications. Arch Intern Med. 2011;171(9):814-22.

10. Dietz RD. The estimation of neighborhood effects in social sciences: an interdisciplinary approach. Soc Sci Res. 2002;31(4):539-75.

11. Cummins S, Curtis S, Diez Roux AV, Macintyre S. Understanding and representing 'place' in health research: a relational approach. Soc Sci Med. 2007;65(9):1825-38.

12. Diez Roux AV. Persistent social patterning of cardiovascular risk: rethinking the familiar. Circulation. 2005;111(23):3020-21.

13 Tonne C, Schwartz J, Mittleman M, Melly S, Suh H, Goldberg R. Longterm survival after acute myocardial infarction is lower in more deprived neighborhoods. Circulation. 2005;111(23):3063-70

14. Isaacs SL, Schroeder SA. Class-the ignored determinant of the nation's health. N Engl J Med. 2004;351(11):1137-42.

15. Hoang C, Kolenic G, Kline-Rogers E, Eagle KA, Erickson SR. Mapping geographic areas of high and low drug adherence in patients prescribed continuing treatment of acute coronary syndrome after discharge. Pharmacotherapy. 2011;31(10):927-33.

16. Egede LE, Gebregziabher M, Hunt KJ, et al. Regional, geographic, and ethnic differences in medication adherence among adults with type 2 diabetes (February). Ann Pharmacother. 2011;Feb 8 [Epub ahead of print].

17. Sargen MR, Hoffstad OJ, Wiebe DJ, Margolis DJ. Geographic variation in pharmacotherapy decisions for U.S. Medicare enrollees with diabetes. J Diabetes Complications. 2012;26(4):301-07.

18. Shacham E, Lian M, Önen NF, Donovan M, Overton ET. Are neighborhood conditions associated with HIV management? HIV Med. 2013;14(10):624-32.

19. Yang C, Wu H, Huang Q, Li Z, Li J. Using spatial principles to optimize distributed computing for enabling the physical science discoveries. Proc Natl Acad Sci USA. 2011;108(14):5498-503.

20, Henry J. Kaiser Family Foundation. Large group insurance market competition. Available at: http://kff.org/other/state-indicator/large-groupinsurance-market-competition/. Accessed October 6, 2014.

21. National Cholesterol Education Program. Third report of the National Cholesterol Education Program Expert Panel on detection, evaluation, and treatment of high blood cholesterol in adults (adult treatment panel III). NIH Publication No. 01-3670. May 2001. Available at: http://www.nhlbi.nih.gov/ guidelines/cholesterol/atp3xsum.pdf. Accessed October 6, 2014.

22. Martin BC, Wiley-Exley EK, Richards S, Domino ME, Carey TS, Sleath BL. Contrasting measures of adherence with simple drug use, medication switching, and therapeutic duplication. Ann Pharmacother. 2009;43(1):36-44

23. Ho PM, Bryson CL, Rumsfeld JS. Medication adherence: its importance in cardiovascular outcomes. Circulation. 2009;119(23):3028-35.

24. Daley DJ, Myint PK, Gray RJ, Deane KH. Systematic review on factors associated with medication non-adherence in Parkinson's disease. Parkinsonism Relat Disord. 2012;18(10):1053-61.

25. Grosset KA, Bone I, Grosset DG. Suboptimal medication adherence in Parkinson's disease. Mov Disord. 2005;20(11):1502-07.

26. Ellis JJ, Erickson SR, Stevenson JG, Bernstein SJ, Stiles RA, Fendrick AM. Suboptimal statin adherence and discontinuation in primary and secondary prevention populations. Should we target patients with the most to gain? J Gen Intern Med. 2004;19(6):638-45.

27. Piette JD, Wagner TH, Potter MB, Schillinger D. Health insurance status, cost-related medication underuse, and outcomes among diabetes patients in three systems of care. Med Care. 2004:42(2):102-09.
28. Sampson RJ, Morenoff JD, Gannon-Rowley T. Assessing "neighborhood effects": social processes and new directions in research. Annu Rev Sociol. 2002;28:443-78.

29. Diez Roux AV. Investigating neighborhood and area effects on health. Am J Public Health. 2001;91(11):1783-89.

30. Chaix B, Lindström M, Rosvall M, Merlo J. Neighborhood social interactions and risk of acute myocardial infarction. J Epidemiol Community Health. 2008;62(1):62-68.

31. Diez-Roux AV, Nieto FJ, Muntaner C, et al. Neighborhood environments and coronary heart disease: a multilevel analysis. Am J Epidemiol. 1997;146(1):48-63.

32. Christakis NA, Fowler JH. The spread of obesity in a large social network over 32 years. N Engl J Med. 2007;357(4):370-79.

33. Berkman LF, Glass T, Brissette I, Seeman TE. From social integration to health: Durkheim in the new millennium. Soc Sci Med. 2000;51(6):843-57.

34. Gallant MP. The influence of social support on chronic illness selfmanagement: a review and directions for research. Health Educ Behav. 2003;30(2):170-95.

35. Johnell K, Råstam L, Lithman T, Sundequist J, Merelo J. Low adherence with antihypertensives in actual practice: the association with social participation-a multilevel analysis. BMC Public Health. 2005:5:17.

36. Chaix B. Geographic life environments and coronary heart disease: a literature review, theoretical contributions, methodological updates, and a research agenda. Annu Rev Public Health. 2009;30:81-105.

37. Patel MR, Greiner MA, DiMartino LD, et al. Geographic variation in carotid revascularization among Medicare beneficiaries, 2003-2006. Arch Intern Med. 2010;170(14):1218-25.

38. Lucas FL, Sirovich BE, Gallagher PM, Siewers AE, Wennberg DE. Variation in cardiologists' propensity to test and treat: is it associated with regional variation in utilization? Circ Cardiovasc Qual Outcomes. 2010;3(3):253-60

39. Panagiotakos DB, Pitsavos C, Leda Matalas A, Chrysohoou C, Stefanadis C. Geographical influences on the association between adherence to the Mediterranean diet and the prevalence of acute coronary syndromes, in Greece: the CARDIO2000 Study. Int J Cardiol. 2005;100(1):135-42.

40. Yeh RW, Normand SL, Wang Y, Barr CD, Dominici F. Geographic disparities in the incidence and outcomes of hospitalized myocardial infarction: does a rising tide lift all boats? Circ Cardiovasc Qual Outcomes. 2012;5(2):197-204.

41. Diez Roux AV, Merkin SS, Arnett D, et al. Neighborhood of residence and incidence of coronary heart disease. N Engl J Med. 2001;345(2):99-106. 42. Sasson C, Magid DJ, Chan P, et al. Association of neighborhood characteristics with bystander-initiated CPR. N Engl J Med. 2012;367(17):1607-15.

43. Gerber Y, Benyamini Y, Goldbourt U, Drory Y; Israel Study Group on First Acute Myocardial Infarction. Neighborhood socioeconomic context and longterm survival after myocardial infarction. Circulation. 2010;121(3):375-83.

44. King WD, Minor P, Ramirez Kitchen CR, et al. Racial, gender, and geographic disparities of antiretroviral treatment among US Medicaid enrollees in 1998. J Epidemiol Community Health. 2008;62(9):798-803.

45. Zerzan JT, Morden NE, Soumerai S, et al. Trends and geographic variation of opiate medication use in state Medicaid fee-for-service programs, 1996 to 2002. Med Care. 2006;44(11):1005-10.

46. Painter JT, Crofford LJ, Talbert J. Geographic variation of chronic opioid use in fibromyalgia. Clin Ther. 2013;35(3):303-11.

47. Czarnecki K, Goranson C, Ellis JA, Vichinsky LE, Coady MH, Perl SB. Using geographic information system analyses to monitor large-scale distribution of nicotine replacement therapy in New York City. Prev Med. 2010;50(5-6):288-96.

48. Brownstein JS, Green TC, Cassidy TA, Butler SF. Geographic information systems and pharmacoepidemiology: using spatial cluster detection to monitor local patterns of prescription opioid abuse. Pharmacoepidemiol Drug Saf. 2010;19(6):627-37. 